\title{
Randomized Controlled Trial on the Provision of the EMDR Integrative Group Treatment Protocol Adapted for Ongoing Traumatic Stress to Female Patients With Cancer-Related Posttraumatic Stress Disorder Symptoms
}

\author{
Ignacio Jarero \\ Martha Givaudan \\ Amalia Osorio \\ Latin American \& Caribbean Foundation for Psychological Trauma Research, Álvaro Obregón, México City, Mexico
}

\begin{abstract}
This randomized controlled trial extended the investigation previously conducted by Jarero et al. (2015) which found that the eye movement desensitization and reprocessing Integrative Group Treatment Protocol adapted for ongoing traumatic stress (EMDR-IGTP-OTS) was effective in reducing posttraumatic stress disorder (PTSD) symptoms related to the diagnosis and treatment of different types of cancer in adult women. The current study sought to determine if the results could be replicated and if the treatment would also be effective in reducing symptoms of anxiety and depression. Participants in treatment ( $N=35)$ and no-treatment control $(N=30)$ groups completed pre, post, and follow-up measurements using the Posttraumatic Stress Disorder Checklist for the Diagnostic and Statistical Manual of Mental Disorders (5th ed.; DSM-5) (PCL-5) and the Hospital Anxiety and Depression Scale (HADS). Data analysis by repeated measures analysis of variance (ANOVA) showed that the EMDR-IGTP-OTS was effective in significantly reducing symptoms of PTSD, anxiety, and depression, with symptoms maintained at 90-day follow-up and with large effect sizes (e.g., $d=1.80$ ). A comparison of the treatment and no-treatment control groups showed significantly greater decreases for the treatment group on symptoms of PTSD, anxiety, and depression. No significant correlation was found when exploring the relationship between scores on the Adverse Life Experiences scale and scores indicating pretreatment severity of PTSD, anxiety, and depression. This study suggests that EMDR-IGTP-OTS may be an efficient and effective way to address cancer-related posttraumatic, depressive, and anxious symptoms.
\end{abstract}

Keywords: eye movement desensitization and reprocessing Integrative Group Treatment Protocol for ongoing traumatic stress (EMDR-IGTP-OTS); posttraumatic stress disorder (PTSD); anxiety; depression; cancer

$\mathbf{T}$

he diagnosis and treatment of cancer is a potentially traumatic experience that may provoke cancer-related posttraumatic stress symptoms (PTSS) that range on a continuum from acute responses postdiagnosis to more severe stress reactions with posttraumatic stress disorder (PTSD), anxiety, and depression symptoms that adversely impact functionality and general well-being (Abbey, Thompson, Hickish, \& Heathcote, 2015; Kangas, 2013). In 2009, the International Psycho-Oncology Society endorsed distress as the sixth vital sign in cancer care (Bultz \& Johansen, 2011). In the World Cancer Declaration, the Union for International Cancer Control (2013) stated that "effective pain control and distress management services will be universally available" (p. 1) was Target \#8 for 2025.

PTSD is a mental condition characterized by a constellation of symptoms that occur following 
exposure to a traumatic event. Those symptoms include intrusion, avoidance of thoughts or reminders of the trauma, negative alterations in cognition and mood, and marked alterations in arousal and reactivity (American Psychiatric Association, 2013). The Diagnostic and Statistical Manual of Mental Disorders (5th ed.; DSM-5; American Psychiatric Association, 2013), notes that a life-threatening illness "is not necessarily considered a traumatic event," but that medical incidents (like cancer) that are "sudden" and "catastrophic events" may qualify (p. 274).

Literature examining PTSD symptomatology among long-term cancer survivors has found rates of lifetime cancer-related PTSD that range from $3 \%$ to $22 \%$, and the presence of comorbid psychiatric illnesses such as major depressive disorder and generalized anxiety disorder that may lead to worse survival outcomes (Andrykowski \& Kangas, 2010; Kangas et al., 2002).

A recent meta-analysis (García-Torres, Alós, \& Pérez-Dueñas, 2015) concluded that psychological and nonpsychological treatments (cognitive behavioral therapy [CBT], mindfulness, stress management training, neuro-emotional therapy, coping skills training, and counseling) available during the last 10 years for PTSD or PTSS in cancer survivors need more evidence to establish their efficacy. Another meta-analysis (Nenova et al., 2013) reviewed the findings of 19 randomized controlled trials testing the efficacy of interventions with CBT components for cancer-related traumatic stress symptoms in adults with cancer and concluded that "there was insufficient evidence from which to draw definitive conclusions about the efficacy of CBT interventions for the treatment of cancer-related traumatic stress symptoms, including PTSD” (pp. 258-259).

\section{EMDR Therapy}

Eye movement desensitization and reprocessing (EMDR) therapy (Shapiro, 2018) is an eight-phase psychotherapeutic comprehensive approach that has been extensively researched and proven effective for the treatment of trauma. According to the World Health Organization (2013), trauma-focused CBT and EMDR therapy are the only psychotherapies recommended for children, adolescents, and adults with PTSD. EMDR therapy has been validated by 38 randomized controlled trials and 8 meta-analyses. See EMDR Research Foundation (2017) for a summary of research findings.

EMDR therapy is guided by the Adaptive Information Processing (AIP) model (Shapiro, 2001, 2018) which posits that the primary source of psychopathology is caused by memories of adverse life experiences that have been inadequately processed and maladaptively stored in a state-specific form. These memories can be triggered by current internal and external stimuli contributing to present dysfunction. EMDR therapy uses a standardized eight-phase procedure, during which clients focus on elements of the disturbing memory, while simultaneously experiencing bilateral stimulation.

\section{EMDR Integrative Group Treatment Protocol Adapted for Ongoing Traumatic Stress}

\section{EMDR-IGTP}

The standard EMDR Integrative Group Treatment Protocol for early intervention (EMDR-IGTP; Artigas $\&$ Jarero, 2014) provides individual EMDR therapy in a group setting. The protocol provides EMDR's eight phases (Shapiro, 2001, 2018) in a group therapy model with an art therapy format (e.g., drawings, symbols). It uses the Butterfly Hug method (BH; Artigas \& Jarero, 2014) as a self-administered bilateral stimulation to process traumatic material. During the session, the client draws a picture or symbol representing the traumatic events, and then focuses on it for an average of 3 minutes while performing the BH method. This process is repeated four times in the session, with the client usually reporting substantial decreases in subjective disturbance at session end. The effectiveness of this protocol has been well documented (e.g., Brown et al., 2017; Jarero \& Artigas, 2010, 2014).

\section{EMDR-IGTP-OTS}

In their case conceptualization, Jarero, Artigas, and Luber (2011) and Jarero and Uribe $(2011,2012)$ assert that for individuals with ongoing external traumatic events in which there is no posttrauma safety window for traumatic memory consolidation, the consolidation of the traumatic memory is prevented, and the patient's memory network remains in a permanent excitatory state as a short-term memory. They view this as a continuum (analogous to the ripple effect of a pebble thrown into a pond), creating a cumulative trauma exposure memory network (Jarero, Amaya, Givaudan, \& Miranda, 2013). They argue that it extends into the present moment, often producing maladaptive/catastrophic concerns about the future or flash-forwards (Logie \& De Jongh, 2014). They believe that this type of ongoing traumatic stress situation requires a different kind of EMDR treatment 
approach than that used for events that have a posttrauma safety period.

Therefore, Jarero et al. (2015) adapted EMDRIGTP in order to treat adolescents (13-17 years old) and adults living with ongoing traumatic stress with no posttrauma safety period for memory consolidation. The targeted memory is selected during Phase 3. Changes in the EMDR-IGTP for ongoing traumatic stress (EMDR-IGTP-OTS) include asking the client to run a mental movie of the whole event from right before the beginning until today, or into the future, and then to identify the hardest, most painful, or most distressing moment. This adaptation was made to allow for the identification, targeting, and processing of the continuum of multiple traumatic experiences faced by this population and not only one target per session. See the work of Jarero, Artigas, Uribe, and García (2016) for a detailed description of the EMDR-IGTP-OTS.

\section{EMDR Therapy Administered to Patients With Cancer}

\section{EMDR Therapy With Individuals}

In a controlled pilot study conducted by Capezzani et al. (2013) for patients with cancer and PTSD, patients treated with EMDR therapy had significant improvement 1-month posttreatment compared to the CBT group. Ten of the 11 patients treated with EMDR therapy lost their PTSD diagnosis compared to only one of the CBT patients. In a nonrandomized study, Faretta, Borsato, Civilotti, Fernandez, and Pagani (2016) evaluated the effectiveness of EMDR therapy compared to a non-trauma-focused CBT intervention in 57 ( 11 males and 46 females) participants with mixed cancer diagnoses that received 12 sessions of 60 minutes each. Those receiving EMDR therapy showed significantly larger posttreatment improvement, compared to the CBT group, on a number of measures, including traumatic stress, depression, and anxiety.

\section{Group Format}

Jarero et al. (2015) conducted a pilot study to evaluate the effectiveness of EMDR-IGTP-OTS in reducing cancer-related PTSD for adult women. EMDR intensive therapy was administered for 3 consecutive days, twice daily, to 24 adult women diagnosed with different types of cancer (cervical, breast, colon, bladder, and skin) who had PTSD symptoms related to their diagnosis and treatment. Treatment outcomes were compared between patients in the active phase of cancer treatment and those in the follow-up phase, with scores on the Short PTSD Rating Interview (SPRINT; Connor \& Davidson, 2001) at pre- and postEMDR treatment and at 30- and 90-day follow-ups. Results showed no difference between groups, with significant improvement in both groups for PTSD symptoms and overall subjective well-being.

\section{Method}

The research protocol was reviewed and approved by the State of Puebla, Mexico, Hospital General del Sur, Eduardo Vazquez Navarro Research Committee with the number 69/ENS/INV/REV/2017 and the Latin American and Caribbean Foundation for Psychological Trauma Research review board to ensure that the research quality of this study partially fulfilled the Revised Gold Standard scale (Maxfield \& Hyer, 2002) items. These included: (a) target symptoms were clearly defined, but without diagnosis; (b) measures were reliable and valid, (c) blind independent evaluators collected posttreatment measures at Times 2 and 3; (d) assessor reliability was checked by M.G.; (e) treatment was manualized; (f) blind random assignment was conducted; $(\mathrm{g})$ treatment fidelity was evaluated by I.J.; (h) no conditions were confounded; (i) multimodal measures were not used; and (j) length of treatment was appropriate for civilian participants with single trauma (five or more sessions). Participation was voluntary and there were four dropouts throughout the study period from the no-treatment control group.

\section{Purpose}

The purpose of the study was to evaluate the effectiveness of the EMDR-IGTP-OTS in reducing PTSD symptoms, depression, and anxiety symptoms related to the diagnosis and treatment of different types of cancer in adult women.

\section{Participants}

This study was conducted in 2017 in the city of Puebla, Mexico, at the Hospital General del Sur, Eduardo Vazquez Navarro. Potential participants were recruited by speaking to each patient personally when they were waiting for their follow-up appointment at the hospital clinic. Those who accepted received an appointment for an interview the same day immediately after their medical appointment in which a qualified research assistant explored whether or not they met the inclusion criteria. A total of 247 potential participants attended the intake interview. 
Inclusion criteria were (a) 18 years or older, (b) diagnosis of cancer, (c) be in the active or follow-up phase of cancer treatment, (d) have Posttraumatic Stress Disorder Checklist for DSM-5 (PCL-5) scores of 30 or higher, (e) not receiving specialized trauma therapy, and (f) not receiving drug therapy for the PTSD symptoms. Exclusion criteria were (a) ongoing self-harm/suicidal or homicidal ideation, (b) diagnosis of psychotic or bipolar disorder, (c) diagnosis of dissociative disorder, (d) organic mental disorder, (e) substance abuse, and (f) significant cognitive impairment (e.g., severe intellectual disability and dementia).

Of the 247 potential participants who attended the intake interview, 177 women did not meet the inclusion/exclusion criteria or were unable to participate in the study. These included 145 potential participants who had PCL-5 scores below 30, and 32 who were unable to participate in the study because they lived in a remote area.

Seventy women with different types of cancer (brain, breast, cervical, uterus, colon, leukemia, lung, ovary, stomach, thyroid gland, medulla, bones, skin, esophagus, liver, and appendix) and PTSD symptoms related to their diagnosis and cancer treatment met the inclusion criteria. Women's age ranged from 26 to 79 years ( $M=47.02$ years). Participants' time since diagnosis varied from 3 months (May, 2017) to 54 months (February, 2013). Participants were randomly selected to treatment group or no-treatment control group using a computer-generated random-number list. Two independent assessors blind to treatment conditions conducted the randomization process to avoid allocation influence.

Participants were contacted by phone to inform them if they belonged to the treatment group or the no-treatment control group. Patients in the no-treatment control group were informed that, for ethical reasons, they would receive the treatment once the first part of the study was concluded. Five patients who had been assigned to the no-treatment control group were not located. There were 35 participants in the treatment group and 30 participants in the no-treatment control group.

\section{Design}

To measure the effect of EMDR-IGTP-OTS in the dependent variables PSTD, anxiety, and depression, this study used a design with a treatment and no-treatment control group. PSTD was measured in three time periods (pre, post, and follow-up); for anxiety and depression we used a pre-post design. The Adverse Childhood Experiences (ACE) questionnaire was applied once at the beginning of the study.

\section{Assessment}

PTSD Checklist for DSM-5. We used the PCL-5 (Weathers et al., 2013) Spanish version provided directly by the National Center for PTSD (NCPTSD) and adapted, with the NCPTSD approval, for the past week instead of the past month, symptoms to conduct research with the high mobility population. It contains 20 items, including three new PTSD symptoms: blame, negative emotions, and reckless or self-destructive behavior. Respondents indicate how much they have been bothered by each PTSD symptom over the past week (rather than the past month), using a 5 -point scale ranging from $0=$ not at all, $1=$ a little bit, $2=$ moderately, $3=$ quite a bit, to $4=$ extremely. Item scores are summed to yield a continuous measure of PTSD symptom severity for symptom clusters and for the whole disorder. According to the National Center for PTSD, a PCL- 5 cutoff point of 33 appears to be a reasonable value to propose until further psychometric work is available.

Hospital Anxiety and Depression Scale. The Hospital Anxiety and Depression Scale (HADS; Zigmond \& Snaith, 1983) is widely used to measure psychological morbidity in cancer patients (Ornelas-Mejorada, Tufiño, \& Sánchez-Sosa, 2011). The HADS is a 14-item self-report scale, using a 4-point Likert scale ranging from 0 to 3. Seven items relate to anxiety and seven to depression. For this study, we used the Spanish adaptation of the HADS for patients with cancer (Rico, Restrepo, \& Molina, 2005). This adaptation showed good internal consistency and validity. An $\alpha$ coefficient of .85 and a splithalf reliability of .8 were found. Cutoff points of 8 and 9 for the anxiety and depression subscales, respectively, showed a favorable sensitivity and specificity in identifying cases of psychological disorder as defined by the psychiatric diagnosis using the DSM-IV-TR (APA, 2000).

ACE Questionnaire. The ACE questionnaire is a 10-item self-report measure developed for the ACE study (Felitti et al., 1998) to identify childhood experiences of abuse and neglect. The ACE score can range from " 0 ," meaning no exposure to the 10 categories of child abuse and trauma investigated by the study, to "10," meaning exposure to all 10 categories. The study found the higher the ACE score, the greater the risk of experiencing poor physical and 
mental health and negative social consequences later in life.

Assessment Times. Treatment and no-treatment control group participants completed the instruments separately in the different measurement moments. By Time 1 (June/July 2017), research assistants collected clinical histories, provided psycho-education, and answered patients' questions related to trauma, PTSD, and EMDR therapy to decrease any possible prejudice against the treatment. Application of instruments for both groups was done after this procedure by research assistants who were not blind to the study. During Time 2 (posttreatment assessment; August 2017) and Time 3 (follow-up assessment 90 days after treatment; November 2017), assessment was conducted for all participants by blind independent assessors, who were not part of the study.

It is important to mention that in the different measurement moments all participants were asked to focus specifically on the worst cancer-related event that currently bothered them the most before answering the PCL-5. A total of 20 research assistants participated in the data collection, all of them licensed EMDR clinicians trained by the second author (M.G.) in instrument administration, general interview techniques, and ethical research conduct.

\section{Treatment}

More frequent scheduling of treatment sessions maximizes PTSD treatment outcomes (Gutner, Suvak, Sloan, \& Resick, 2016). In this study, intensive EMDR therapy (Bongaerts, Van Minnen, $\&$ de Jongh, 2017; Grey, 2011; Jarero et al., 2015; Lobenstine \& Courtney, 2013; Wesson \& Gould, 2009) was provided because many participants traveled to the hospital from long distances and only stayed there for a limited time. This intensive format allowed patients to complete the full course of treatment in a short period.

Therapists. EMDR-IGTP-OTS was administered by six licensed EMDR clinicians formally trained in the protocol administration. One was a certified EMDR therapist and five were certified EMDR therapists in training. Fidelity was assessed by the supervisor (I.J.).

Treatment. In August 2017, the treatment group participants received six treatment sessions during 2 consecutive days, three times daily in a setting inside the hospital where they received cancer treatment or follow-up. There was one therapy group with 35 participants. EMDR-IGTP-OTS treatment focused only on the cumulative trauma memories related to the oncological disease and did not address any previous traumatic events. The first group session lasted 1 hour and 40 minutes. The next group sessions had an average duration of 50 minutes since they started in Phase 3 of the protocol, as it was not necessary to repeat Phases 1 and 2. Participants received an average of six treatment hours in total. There were no dropouts in the treatment group. No adverse effects were reported during treatment or at 90-day follow-up. Treatment fidelity was fulfilled by therapists' strict observance to all steps of the scripted protocol (Jarero et al., 2016) while they were assessed by the supervisor (I.J.).

\section{Data Analysis}

Analysis of variance (ANOVA) for repeated measurements was carried out with different time points (three time points for PCL-5 and two time points for anxiety and depression) and two groups (treatment $=35$ subjects; no-treatment control $=26$ subjects) as independent variables and PTSD (PCL-5), anxiety, and depression as dependent variables. Results of the $\beta^{2}$ variables are presented. In addition, Cohen's $d$ was used to measure the size of the significant effects of the study. Spearman's correlations were obtained separately for the treatment and control group.

\section{Results}

\section{Patient Flow}

The study began with 70 patients randomly assigned to the treatment group or the no-treatment control group. Of the 35 patients assigned to the control group, five could not be located for treatment allocation. Then another four patients in the no-treatment control group dropped out, leaving 26 participants in this group. None of the 35 participants in the treatment group dropped out of the study.

\section{Treatment Outcome}

PTSD (PCL-5). Data analysis by repeated measures ANOVA revealed a significant effect for time $(F[2,118]$ $\left.=107.41, p<.001, \eta_{\mathrm{p}}{ }^{2}=.645\right)$, a significant effect for group $\left(F[1,59]=22.00, p<.001, \eta_{\mathrm{p}}{ }^{2}=.272\right)$, and a significant interaction between time and group $(F[2$, $118]=30.66, p<.001, \eta_{\mathrm{p}}{ }^{2}=.342$ ). Post hoc comparisons using the Bonferroni correction indicated significant differences among the means. In the treatment group, mean scores showed a significant decrease between Time 1 and Time 2, $t(34)=15.13, p<.05, d$ $=1.80$. There was also a significant decrease between Time 1 and Time 3, $t(34)=12.91, p<.05, d=1.74$. In 
TABLE 1. Mean Scores and Standard Deviations for Treatment and No-Treatment Group on Pretest, Posttest, and Follow-Up Measurements

\begin{tabular}{|c|c|c|c|c|c|c|}
\hline \multirow[b]{2}{*}{ Variable } & \multicolumn{2}{|c|}{ Time 1} & \multicolumn{2}{|c|}{ Time 2} & \multicolumn{2}{|c|}{ Time 3} \\
\hline & $M$ & $S D$ & $M$ & $S D$ & M & $S D$ \\
\hline \multicolumn{7}{|c|}{ Posttraumatic stress (PCL-5) } \\
\hline Treatment group & 44.89 & 10.26 & 20.51 & 8.80 & 17.89 & 11.67 \\
\hline No-treatment group & 43.85 & 11.19 & 37.19 & 12.89 & 35.088 & 9.79 \\
\hline \multicolumn{7}{|l|}{ Anxiety } \\
\hline Treatment group & 12.74 & 3.70 & & & 7.34 & 4.39 \\
\hline No-treatment group & 11.65 & 4.33 & & & 12.19 & 3.68 \\
\hline \multicolumn{7}{|l|}{ Depression } \\
\hline Treatment group & 8.94 & 3.47 & & & 5.54 & 4.22 \\
\hline No-treatment group & 7.81 & 3.60 & & & 10.84 & 3.72 \\
\hline
\end{tabular}

Note. PCL-5 = Posttraumatic Stress Disorder Checklist for DSM-5.

the control group, mean scores showed a significant decrease between Time 1 and Time 2, $t(25)=3.87, p$ $<.05, d=.39$. There was also a significant decrease between Time 1 and Time 3, $t(25)=5.31, p<.05, d=$ .59. Comparison between groups did not show significant differences for Time 1. For Time 2 and Time 3 significant differences among the groups were found, $t(59)=-6.00, p<.05, d=1.06$ and $t(59)=6.074, p<$ $.05, d=1.12$, respectively. See Table 1 and Figure 1 .

Anxiety. Repeated measures ANOVA showed a significant effect for time $(F[1,59]=12.86, p<$ $\left..001, \eta_{\mathrm{p}}{ }^{2}=.179\right)$, a significant effect for group $(F[1,59]$ $\left.=5.56, p<.05, \eta_{\mathrm{p}}{ }^{2}=.086\right)$, and a significant interaction effect between time and group $(F[1,59]=19.19$, $\left.p<.001, \eta_{\mathrm{p}}{ }^{2}=.245\right)$. Post hoc comparisons using the
Bonferroni correction indicated significant differences for treatment group, $t(34)=5.65, p<.05, d=.94$. No significant differences were observed in the no-treatment control group. Comparison between groups did not show significant differences between groups for Time 1; by Time 3 significant differences among the groups were found, $t(59)=-4.56, p<.05, d=.84$. See Table 1 and Figure 2.

Depression. The results of the repeated measures ANOVA showed a significant effect for group, $F(1$, $59)=6.50, p>.05, \eta_{\mathrm{p}}{ }^{2}=.099$, and a significant effect for the interaction between time and group, $F(1,59)$ $=35.79, p<.001, \eta_{\mathrm{P}}{ }^{2}=.378$. Post hoc comparisons using the Bonferroni correction indicated significant differences for treatment group, $t(34)=5.39, p<.05$,

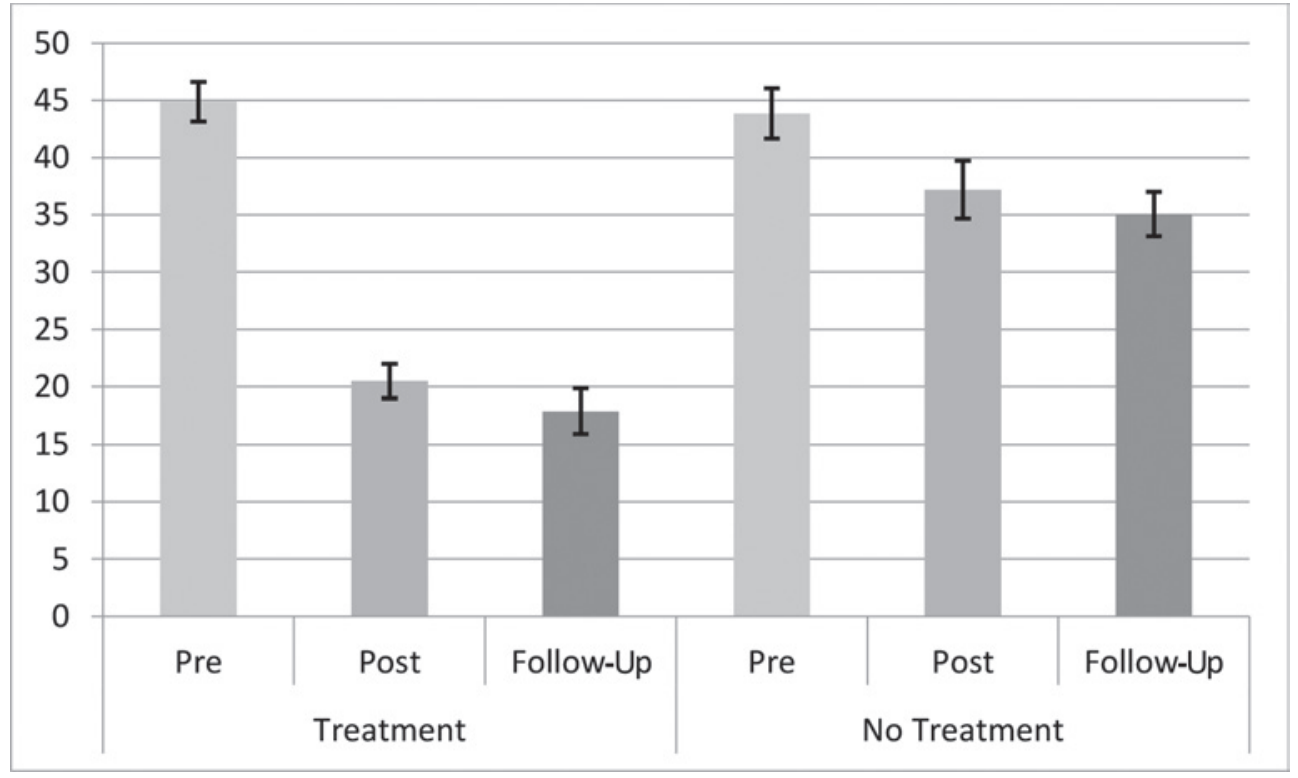

FIGURE 1. Mean scores and standard error for PCL-5 by time and group. 


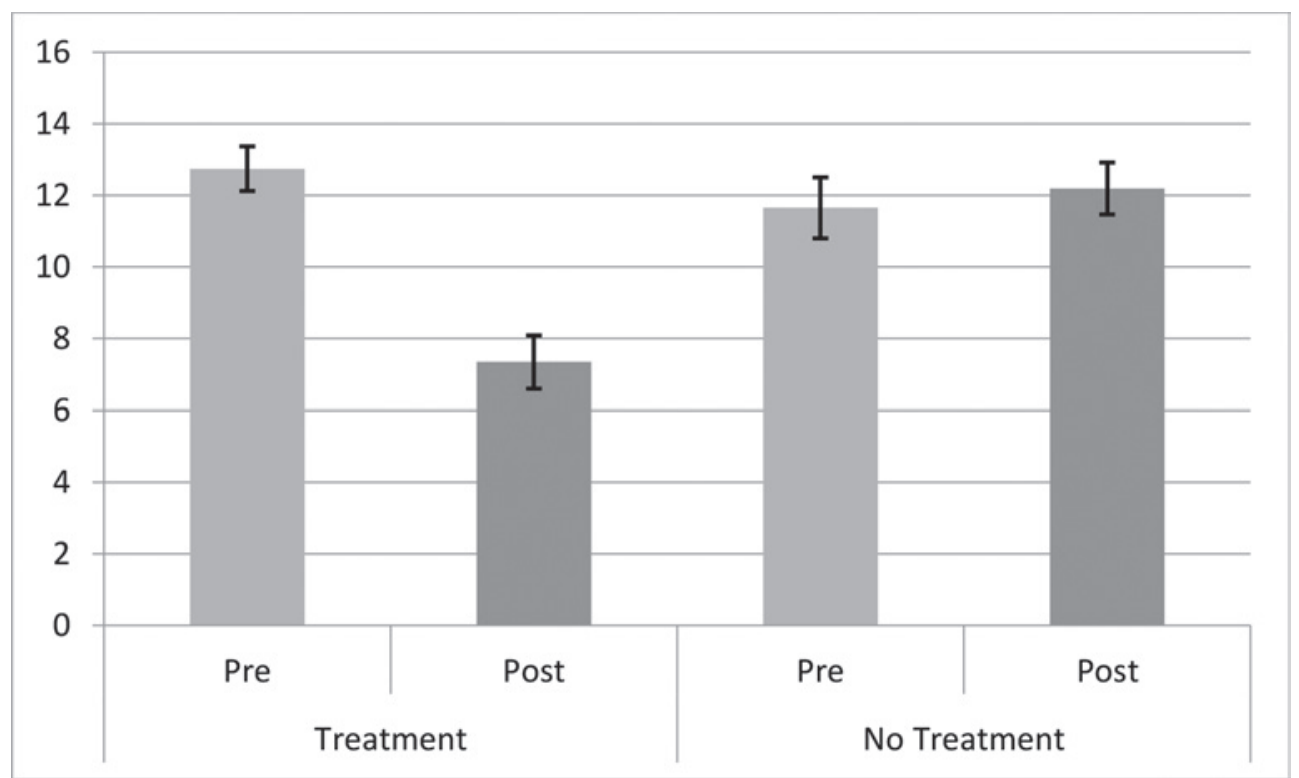

FIGURE 2. Mean scores and standard error for anxiety by time and group.

$d=.62$. The no-treatment control group increased significantly from Time 1 to Time $3, t(25)=3.31, p$ $<.05, d=-.58$. Comparison between groups did not show significant differences between groups for Time 1; by Time 3 significant differences among the groups were found, $t(59)=-5.19, p<.05, d=-0.94$. See Table 1 and Figure 3.

ACE Scale. Scores on the ACE scale for the 65 participants varied from 0 to 9 with an average of 3.41 and with similar means for each group $(M=3.26, S D$ $=2.38$ for treatment group and $M=3.03, S D=2.85$ for control group). Analyses showed that there were no significant correlations between scores on the ACE scale and the pretreatment PCL-5 scores $(r=.135, p=$ $.13)$, the pretreatment anxiety $(r=.098, p=.45)$, and depression scores $(r=.024, p=.85)$.

\section{Discussion}

The treatment group participants reprocessed their cancer-related pathogenic memories (Centonze, Siracusane, Calabresi, \& Bernardi, 2005) with efficiency (only six sessions), efficacy (symptoms reduction), and without associations with past memories just as if they were reprocessing a recent event, even though time since diagnosis varied from over 4 years

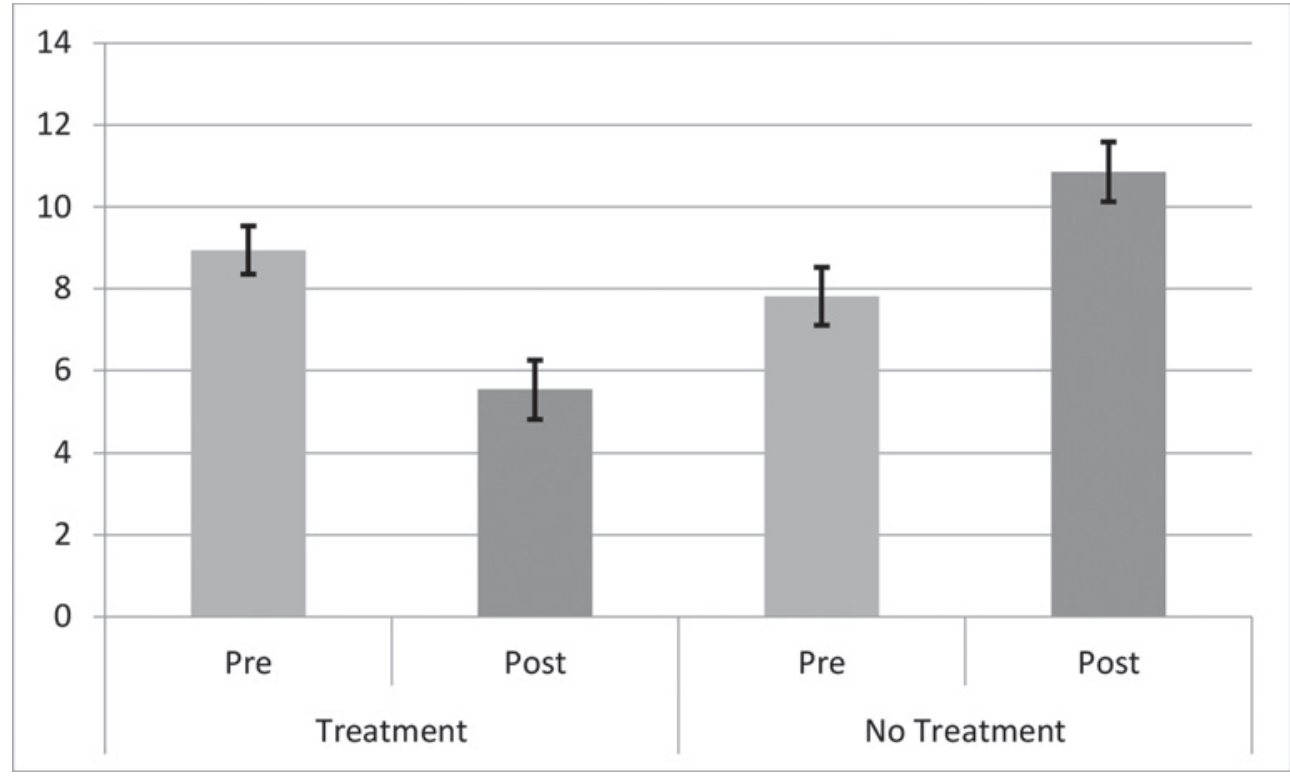

FIGURE 3. Mean scores and standard error for depression by time and group. 
to just 3 months. This clinical observation is consistent with the results from Jarero et al's. (2015) pilot study with a similar population and with the previously mentioned AIP-based case conceptualization. Perhaps Rosenblum, Dockstader, and Martin (2017) assertion could explain part of this clinical phenomenon: "Because EEI (early EMDR interventions) does not delve into the past, it is typically six or fewer sessions and, yet it provides strong, significant, enduring, symptom reduction" (p. 7).

With respect to anxiety and depression, there were significant differences between the treatment group and the no-treatment control at follow-up. Anxiety decreased significantly from pretest to follow-up in the treatment group and no changes were observed for the no-treatment control group. Depression decreased significantly in the treatment group from pretest to follow-up and increased significantly in the no-treatment control group. These results indicate that the EMDR-IGTP-OTS was not only effective in reducing the PTSD symptoms but also had a clear effect in the reduction of anxiety and depression.

\section{Changes in the No Treatment Control Group}

It is interesting to note that there was a statistically significant decrease in the no-treatment control group's PCL- 5 scores from pretest to posttest and from pretest to follow-up, with a small effect size (Cohen's d). Nevertheless, even though this group showed some improvement, there was a significant difference between their scores and those of the treatment group at Times 2 and 3, with the treatment group showing significantly larger effects (see Figure 1).

In addition to noting the large difference between the two groups, we consider that the clinical interpretation can be useful to explain these results. Evidence for the PCL for DSM-IV suggests that a 10- to 20-point change in PCL-5 represents clinically significant change (Blevins, Weathers, Davis, Witte, \& Domino, 2015). Applying this criterion, the treatment group (with a mean decrease of 24.4 points) experienced both a statistically significant decrease with a large effect size and a clinically significant decrease, while the no-treatment control group showed a statistically significant decrease in PCL-5 scores, with a small effect size and no clinically significant change ( 8.3 points).

Another hypothesis - based on the study of Liu, Zhang, Jiang, and $\mathrm{Wu}$ (2017) with ovarian cancer survivors and the study of Andrykowski, Cordova, McGrath, Sloan, and Kenady (2000) with breast cancer survivors, which found significant fluctuations (waxing and waning symptoms course) in PTSD symptoms within individuals - is that greater social support, experience of fewer traumatic stressors prior to cancer diagnosis, hope, and resilience through time could be associated with the decrease of PTSD symptoms. Future longitudinal research on PTSD symptoms' fluctuation with multiple cancer-type survivors is recommended. Another possibility is that the psychoeducational interview session had a therapeutic effect, as the research assistants were empathic and supportive. The participants reported that it was the first time they were asked about their psycho-emotional symptoms and said that the psycho-education helped them to understand those symptoms as a by-product of their cancer diagnosis and treatment. Some women reported that this released them from the distress of believing they were crazy or that the cancer was their fault.

\section{The ACE Scale}

The number of adverse childhood experiences among the 65 participants varied from 0 to 9 with an average of 3.41 and with similar means for each group. There were no correlations between the ACE score and the pretreatment scores of PCL-5, anxiety, and depression. This suggests that ACE does not seem to be a risk factor for the traumatic anxious or depressive symptoms of women dealing with stress related to cancer diagnosis and treatment. It indicated that the severity of their symptoms was unrelated to the number of childhood adverse experiences and suggests that the symptoms were primarily related to their cancer diagnosis and treatment. This appears to show the ongoing nature of the traumatic stress experienced by the participants, even though the time of diagnosis for some was several years earlier.

\section{EMDR-IGTP-OTS Advantages}

This protocol has all the standard EMDR-IGTP advantages and allows for the identification, targeting, and processing of the continuum of multiple traumatic experiences faced by cancer-related PTSD populations who are experiencing ongoing traumatic stress and not only one target per session. Individual EMDR treatment can be provided to small or large groups of patients in an intensive EMDR treatment modality so that patients can receive efficient and effective treatment. With this protocol, clinicians could treat groups of patients who have been through the same type of ongoing or prolonged traumatic events or circumstances. These include victims of constant violence, at-risk personnel, people undergoing life-changing experiences with ongoing traumatic stress or extreme stressors, or people with diverse 
ongoing trauma histories with similar circumstances in common.

As a cross-cultural treatment, EMDR-IGTP-OTS reduces cultural resistance to treatment, even to members of reticent cultures, because it is minimally intrusive, and does not require creating a narrative of the traumatic experience, verbal or written disclosure of details, prolonged reliving of traumatic experiences, or homework. Relying on drawings presents a special advantage to provide culturally sensitive and effective treatment for patients who struggle to connect to their cognitive states or feel guilty or ashamed; they may be more comfortable expressing their emotional distress through drawing. Also, drawings are used for effective reprocessing with patients with lower levels of literacy (Shapiro, 2016) like the participants in this study.

\section{Treatment Tolerance}

The accumulation of dysfunctional stored memories increases the likelihood of PTSD (McFarlane, 2009, 2010b, 2010a). We believe that the use of self-administered bilateral stimulation (BLS) that incorporates eye movements and/or eye movements and tapping in a group setting with a population with ongoing traumatic stress could provoke abreactions that would be difficult to manage. Therefore, participants in this study used the BH (Artigas \& Jarero, 2014) as a self-administered bilateral stimulation method to process traumatic material. During the $\mathrm{BH}$, patients are instructed to stop when they feel in their body that it has been enough. This instruction allows for enough sets of BLS for processing the traumatic material and helps to regulate the stimulation to maintain the patients in their window of tolerance (Ogden, Minton, \& Pain, 2006; Siegel, 1999) allowing for appropriate reprocessing.

\section{EMDR-IGTP-OTS Limitations}

During Phase 3, participants are not asked about negative or positive cognitions as in the standard EMDR protocol. Phase 5 (Installation) of the standard EMDR protocol cannot be conducted for the following reasons: each participant may have a different Subjective Units of Disturbance (SUD) score, blocking beliefs, or have different timing for processing and reach an ecological level of disturbance. At this stage of the protocol, we work on a future vision to identify adaptive or nonadaptive drawings and cognitions that are helpful in the evaluation of the participant at the end of the protocol administration. Present and future template prongs of the standard protocol are not covered in this protocol. Authors' clinical observations showed that after six administrations, an average of $8 \%$ of participants will need additional treatment. They recommend the EMDR Protocol for Recent Critical Incidents and ongoing traumatic stress (EMDR-PRECI; Jarero et al., 2011) for individual treatment.

\section{Future Directions and Recommendations}

According to Maxfield (2016), "EMDR therapy is uniquely positioned to be an optimal treatment for the distress experienced by patients with cancer and other distressing medical illness" (p. 136). We believe that when cancer-related PTSD symptoms are untreated, medical and psychiatric morbidity could increase. Therefore, the implementation of more randomized controlled research on EMDR therapy-based early interventions for individuals and groups specially designed for acute trauma and/or ongoing traumatic stress aimed at taking care of psychopathology reported by patients with cancer is an area of fundamental interest.

It is important for clinicians and researchers to be aware of the nature of cancer as a unique ongoing traumatic stressor that could produce psychological suffering and cumulative trauma exposure memory networks in a substantial number of patients, with triggers for cancer-related PTSD symptoms in the medical setting during routine treatment procedures and follow-up appointments. EMDR-IGTP-OTS is a psychotherapy protocol as well as a screening tool for EMDR therapy individual treatment for those who require additional support after completing the group treatment. Its use-when facing a large number of patients needing treatment, like the cancer-related PTSD populationcould be an auspicious treatment to improve the cancer patient's psychological recovery.

\section{References}

Abbey, G., Thompson, S. B., Hickish, T., \& Heathcote, D. (2015). A meta-analysis of prevalence rates and moderating factors for cancer-related post-traumatic stress disorder. Psycho-Oncology, 24(4), 371-381. http: / / dx.doi. org/10.1002/pon.3654

American Psychiatric Association. (2000). Diagnostic and statistical manual of mental disorders (4th ed.). Washington, DC: Author.

American Psychiatric Association. (2013). Diagnostic and statistical manual of mental disorders (5th ed.). Arlington, VA: Author.

Andrykowski, M. A., Cordova, M. J., McGrath, P. C., Sloan, D. A., \& Kenady, D. E. (2000). Stability and change in posttraumatic stress disorder symptoms following breast cancer treatment: A 1-year follow-up. Psycho-Oncology, 9(1), 69-78. http://dx.doi.org/10.1002/(SICI)10991611(200001 / 02)9:1\&lt;69::AID-PON439\&gt;3.0.CO;2-R 
Andrykowski, M. A., \& Kangas, M. (2010). Posttraumatic stress disorder associated with cancer diagnosis and treatment. In J. C. Holland, W. S. Breitbart, P. B. Jacobsen, M. S. Lederberg, M. J. Loscalzo, \& R. McCorklen (Eds.), Oxford textbook of psycho-oncology (2nd ed., pp. 348-357). New York, NY: Oxford University Press.

Artigas, L., \& Jarero, I. (2014). The Butterfly Hug. In M. Luber (Ed.), Implementing EMDR early mental health interventions for man-made and natural disasters: Models, scripted protocols, and summary sheets (pp. 127-130). New York, NY: Springer Publishing.

Blevins, C. A., Weathers, F. W., Davis, M. T., Witte, T. K., \& Domino, J. L. (2015). The Posttraumatic Stress Disorder Checklist for DSM-5 (PCL-5): Development and initial psychometric evaluation. Journal of Traumatic Stress, 28(6), 489-498. http: / / dx.doi.org/10.1002/jts.22059

Bongaerts, H., Van Minnen, A., \& de Jongh, A. (2017). Intensive EMDR to treat patients with complex posttraumatic stress disorder: A case series. Journal of EMDR Practice and Research, 11(2), 84-95. http:/ / dx.doi.org/10. 1891/1933-3196.11.2.84

Brown, R. C., Witt, A., Fegert, J. M., Keller, F., Rassenhofer, M., \& Plener, P. L. (2017). Psychosocial interventions for children and adolescents after man-made and natural disasters: A meta-analysis and systematic review. Psychological Medicine, 47(11), 1893-1905. http://dx.doi.org/ 10.1017 / S0033291717000496

Bultz, B. D., \& Johansen, C. (2011). Screening for distress, the 6th vital sign: Where are we, and where are we going? Psycho-Oncology, 20(6), 569-571. http://dx.doi. org/10.1002/ pon.1986

Capezzani, L., Ostacoli, L., Cavallo, M., Carletto, S., Fernandez, I., Solomon, R., . . .Cantelmi, T. (2013). EMDR and CBT for cancer patients: Comparative study of effects on PTSD, anxiety, and depression. Journal of EMDR Practice and Research, 7(3), 134-143. http://dx. doi.org/10.1891/1933-3196.7.3.134

Centonze, D., Siracusane, A., Calabresi, P., \& Bernardi, G. (2005). Removing pathogenic memories. Molecular Neurobiol, 32, 123-132.

Connor, K. M., \& Davidson, J. R. (2001). SPRINT: A brief global assessment of post-traumatic stress disorder. International Clinical Psychopharmacology, 16(5), 279-284. http: / / dx.doi.org/ 10.1097 / 00004850-200109000-00005

EMDR Research Foundation. (2017). Research list. Retrieved from http://www.emdrresearchfoundation. org/ research-list

Faretta, E., Borsato, T., Civilotti, C., Fernandez, I., \& Pagani, M. (2016). EMDR and CBT: A comparative clinical study with oncological patients. Journal of EMDR Practice and Research, 10(3), 215-227. http: / / dx.doi.org/ 10.1891/1933-3196.10.3.215

Felitti, V. J., Anda, R. F., Nordenberg, D., Williamson, D. F., Spitz, A. M., Edwards, V., . . .Marks, J. S. (1998). Relationship of childhood abuse and household dysfunction to many of the leading causes of death in adults. The Adverse Childhood Experiences (ACE)
Study. American Journal of Preventative Medicine, 14(4), 245-258.

García-Torres, F., Alós, F. J., \& Pérez-Dueñas, C. (2015). El Trastorno por Estrés Postraumático en los supervivientes al cáncer: Una revisión de los tratamientos psicológicos disponibles. Psicooncología, 12(2-3), 293-301.

Grey, E. (2011). A pilot study of concentrated EMDR: A brief report. Journal of EMDR Practice and Research, 5(1), 14-24. http: / / dx.doi.org/10.1891/1933-3196.5.1.14

Gutner, C. A., Suvak, M. K., Sloan, D. M., \& Resick, P. A. (2016). Does timing matter? Examining the impact of session timing on outcome. Journal of Consulting and Clinical Psychology, 84(12), 1108-1115. http://dx.doi. org/10.1037/ ccp0000120

Jarero, I., Amaya, C., Givaudan, M., \& Miranda, A. (2013). EMDR individual protocol for paraprofessional use: A randomized controlled trial with first responders. Journal of EMDR Practice and Research, 7(2), 55-64. http://dx. doi.org/10.1891/1933-3196.7.2.55

Jarero, I., \& Artigas, L. (2010). The EMDR integrative group treatment protocol: Application with adults during ongoing geopolitical crisis. Journal of EMDR Practice and Research, 4(4), 148-155. http://dx.doi.org/10. 1891/1933-3196.4.4.148

Jarero, I., Artigas, L., \& Luber, M. (2011). The EMDR protocol for recent critical incidents: Application in a disaster mental health continuum of care context. Journal of EMDR Practice and Research, 5(3), 82-94. http: / / dx.doi.org/10.1891/1933-3196.5.3.82

Jarero, I., Artigas, L., Uribe, S., \& Miranda, A. (2014). EMDR Therapy humanitarian trauma recovery interventions in Latin America and the Caribbean. Journal of EMDR Practice and Research, 8(4), 260-268. http: / / dx. doi.org/10.1891/1933-3196.8.4.260

Jarero, I., Artigas, L., Uribe, S., \& García, L. E. (2016). The EMDR Integrative Group treatment protocol for patients with cancer. Journal of EMDR Practice and Research, 10(3), 199-207. http://dx.doi.org/10.1891/ 1933-3196.10.3.199

Jarero, I., Artigas, L., Uribe, S., García, L. E., Cavazos, M. A., \& Givaudan, M. (2015). Pilot research study on the provision of the EMDR Integrative Group Treatment Protocol with female cancer patients. Journal of EMDR Practice and Research, 9(2), 98-105.

Jarero, I., \& Uribe, S. (2011). The EMDR protocol for recent critical incidents: Brief report of an application in a human massacre situation. Journal of EMDR Practice and Research, 5(4), 156-165. http:/ / dx.doi.org/10.1891/ 1933-3196.5.4.156

Jarero, I., \& Uribe, S. (2012). The EMDR protocol for recent critical incidents: Follow-up report of an application in a human massacre situation. Journal of EMDR Practice and Research, 6(2), 50-61. http://dx.doi.org/10.1891/19333196.6.2.50

Kangas, M. (2013). DSM-5 trauma and stress related disorders: Implications for screening for cancer related stress. 
Frontiers in Psychiatry, 4, 122. http://dx.doi.org/10. 3389 / fpsyt.2013.00122

Kangas, M., Henry, J. L., \& Bryant, R. A. (2002). Posttraumatic stress disorder following cancer: A conceptual and empirical review. Clinical Psychology Review, 22(4), 499-524.

Liu, C., Zhang, Y., Jiang, H., \& Wu, H. (2017). Association between social support and post-traumatic stress disorder symptoms among Chinese patients with ovarian cancer: A multiple mediation model. PLoS ONE, 12(5), e0177055. http://dx.doi.org/10.1371/journal. pone. 0177055

Lobenstine, F., \& Courtney, D. (2013). A case study: The integration of intensive EMDR and ego state therapy to treat comorbid posttraumatic stress disorder, depression, and anxiety. Journal of EMDR Practice and Research, 7(2), 65-80. http://dx.doi.org/10.1891/1933-3196.7.2. 65

Logie, R. D. J., \& De Jongh, A. (2014). The "flashforward" procedure: Confronting the catastrophe. Journal of EMDR Practice and Research, 8(1), 25-32. http://dx.doi. org/10.1891/1933-3196.8.1.25

Maxfield, L. (2016). EMDR therapy and psycho-oncology. Journal of EMDR Practice and Research, 10(3), 135-137. http: / / dx.doi.org/10.1891/1933-3196.10.3.135

Maxfield, L., \& Hyer, L. (2002). The relationship between efficacy and methodology in studies investigating EMDR treatment of PTSD. Journal of Clinical Psychology, 58(1), 23-41. http: / / dx.doi.org/10.1002/jclp.1127

McFarlane, A. C. (2009). The duration of deployment and sensitization to stress. Psychiatric Annals, 39(2), 81-88. http: / / dx.doi.org/10.3928/00485713-20090201-05

McFarlane, A. C. (2010a). Abstract to plenary presentation Paper presented at EMDR Europe Annual Conference, Hamburg, Germany.

McFarlane, A. C. (2010b). The long-term costs of traumatic stress: Intertwined physical and psychological consequences. World Psychiatry, 9(1), 3-10. http: / dx.doi.org/ 10.1002/j.2051-5545.2010.tb00254.x

Nenova, M., Morris, L., Paul, L., Li, Y., Applebaum, A., \& DuHamel, K. (2013). Psychosocial interventions with cognitive-behavioral components for the treatment of cancer-related traumatic stress symptoms: A review of randomized controlled trials. Journal of Cognitive Psychotherapy, 27(3), 258-284. http://dx.doi.org/10.1891/ 0889-8391.27.3.258

Ogden, P., Minton, K., \& Pain, C. (2006). Trauma and the body: A sensorimotor approach to psychotherapy. New York, NY: Norton.

Ornelas-Mejorada, R. E., Tufiño, M. A., \& Sánchez-Sosa, J. J. (2011). Ansiedad y depresión en mujeres con cáncer de mama en radioterapia: Prevalencia y factores asociados. Acta de Investigación Psicológica, 1(3), 401-414.

Rico, J. L., Restrepo, M., \& Molina, M. (2005). Adaptación y validación de la escala hospitalaria de ansiedad y depresión (HAD) en una muestra de pacientes con cáncer del Instituto Nacional de Cancerología de Colombia. Avances en Medición, 3, 73-86.

Rosenblum, R. E., Dockstader, D. J., \& Martin, S. A. (2017). EMDR, community psychology and innovative applications of a trauma recovery network as a toll of social change. Journal of EMDR Practice and Research, 11(4), 206-216. http: / / dx.doi.org/10.1891/1933-3196.11.4.206

Shapiro, F. (2001). Eye movement desensitization and reprocessing. Basic principles, protocols, and procedures (2nd ed.). New York, NY: Guilford Press.

Shapiro, F. (2016). Clinician's corner: EMDR therapy. International Society for Traumatic Stress Studies. Stress Points. Retrieved from http://www.istss.org/educationresearch / traumatic-stresspoints / 2016-april/clinician-scorner-emdr-therapy.aspx

Shapiro, F. (2018). Eye movement desensitization and reprocessing (EMDR) therapy: Basic principles, protocols, and procedures (3rd ed.). New York, NY: Guilford Press.

Siegel, D. J. (1999). The developing mind: How relationships and the brain interact to shape who we are. New York, NY: Guilford Press.

Union for International Cancer Control. (2013). World cancer declaration. Retrieved from http://www.uicc. org/who-we-are/world-cancer-declaration

Weathers, F. W., Litz, B. T., Keane, T. M., Palmieri, P. A., Marx, B. P., \& Schnurr, P. P. (2013). The PTSD checklist for DSM-5 (PCL-5). Scale available from the National Center for PTSD. Retrieved from www.ptsd.va.gov

Wesson, M., \& Gould, M. (2009). Intervening early with EMDR on military operations: A case study. Journal of EMDR Practice and Research, 3(2), 91-97. http:/ / dx.doi. org/10.1891/1933-3196.3.2.91

World Health Organization. (2013). Guidelines for the managements of conditions specifically related to stress. Geneva, Switzerland: Author.

Zigmond, A. S., \& Snaith, R. P. (1983). The hospital anxiety and depression scale. Acta Psychiatrica Scandinavica, 67(6), 361-370. http://dx.doi.org/10.1111/j.1600-0447. 1983.tb09716.x

Acknowledgments. The authors would like to thank Ana Gabriela Arriaga, Claudia Badillo, Yael Becker, Diana W. Chávez, Christiane Chedraui, Paola Eunice Díaz-Rivera, Carolina Domínguez, Nancy Farah Escalante, María Diana Espinosa, Myriam Sandra del Carmen Jiménez, María José Millán, Pía Morfín, Katya Muñoz, Úrsula Mariana Ocampo, Giselle Ortiz, María Cristina Pérez, María Leticia Picazo, Verónica Quintana, Silvia Patricia Ramírez, María Pía Rodríguez, Susana Schneider, Kelly Smyth-Dent, Gabriela Tirado, Susana Uribe, Claudia Varela, Alma Belén Vergara.

Correspondence regarding this article should be directed to Ignacio Jarero, $\mathrm{PhD}$, EdD, Latin American \& Caribbean Foundation for Psychological Trauma Research, Boulevard de la Luz 771, Jardines del Pedregal, Álvaro Obregón, México City, Mexico 01900. E-mail: nacho@amamecrisis. com.mx 